\title{
Capacidades para la agencia en egresados del telebachillerato comunitario
}

\section{Capabilities for agency in graduates at the telebachillerato comunitario (a Mexican high-school service)}

JosÉ FRANCISCo Alanís JimÉnEZ*

El objetivo de este artículo es revisar los casos de 25 egresados de telebachillerato comunitario en el estado de Morelos a fin de rastrear sus principales aprendizajes no académicos y valorar su relevancia en términos de agencia desde una perspectiva de desarrollo humano. La vía metódica partió de la perspectiva fenomenológica, que se ajustó a un estudio de casos múltiples con unidades incrustadas según las orientaciones de Yin (2018). La aproximación teórica se apoyó en conceptos de capacidades y agencia, adaptados a procesos educativos con base en aportaciones de Sen (2019), Nussbaum (2012) y Appadurai (2013). Para el análisis, se retomó la codificación temática propuesta por Flick (2017). Las conclusiones resaltan la importancia de los aprendizajes no académicos para el desarrollo de la agencia y aportan propuestas prescriptivas para potencializar aún más sus beneficios en este servicio educativo de nivel medio superior.

The main objective of this text is to review the cases of 25 pre-university graduates from the "telebachillerato comunitario" (community distance-high school), in Morelos State, in order to track their main non-academic learning accomplishments and describe their relevance in terms of agency, within a human development perspective. The method departs from a phenomenological perspective and then engages in a multiple case study with embedded units, according to Yin (2018)'s guidelines. Theoretical approach describes notions of agency and capabilities adapted to education, considering Sen (2019), Nussbaum (2012) and Appadurai (2013), while data analysis uses the thematic codification process proposed by Flick (2017). The conclusions highlight the importance of such learning to develop agency; they also provide a prospective view in order to further enhance the benefits of this high school educational service.
Palabras clave:

educación comunitaria, educación y desarrollo, autonomía, aprendizaje activo, transformación personal

\section{Keywords:}

community education, education and development, autonomy, experiential learning, personal transformation

Recibido: 13 de febrero de 2020 | Aceptado para su publicación: 16 de julio de 2020 | Publicado: 5 de agosto de 2020

Recuperado de: https://sinectica.iteso.mx/index.php/SINECTICA/article/view/1084 doi: 10.31391/S2007-7033(2020)0055-005

\footnotetext{
* UNAM, Becario del programa de becas posdoctorales en la UNAM, Centro Regional de Investigaciones Multidisciplinarias. Asesorado por la doctora Carlota Guzmán Gómez. Doctor en Educación por la UAEM, Morelos. Líneas de investigación: telebachilleratos comunitarios y formación docente. Correo electrónico: picoalanis@gmail.com / https://orcid. org/: 0000-0002-3092-3397
} 


\section{INTRODUCCIÓN}

n 2015, la Organización de las Naciones Unidas para la Educación, la Ciencia y 4 la Cultura (Unesco) propuso replantear la educación, en continuidad con los informes Faure y Delors, los cuales indican que cabe entender el conocimiento como "el modo en que los individuos y las sociedades dan un sentido a la experiencia"; que el aprendizaje sería el "proceso necesario para adquirir ese conocimiento. Es a la vez el proceso y el resultado de ese proceso"; y que "educación significa aquí un aprendizaje que se caracteriza por ser deliberado, intencionado, con un fin determinado y organizado" (Unesco, 2015, p. 17). Considerando la manera en que nos adentramos en un nuevo milenio, también especifican: "Apoyar y aumentar la dignidad, la capacidad y el bienestar de la persona humana en relación con los demás y la naturaleza debe ser la finalidad esencial de la educación en el siglo XXI" (p. 36). No sería necesario replantear la educación si el rumbo correcto se pudiera mantener con facilidad y si no se hubiera errado ya en algunos aspectos fundamentales. Vale la pena revisar nuestros modelos educativos a la luz de estas orientaciones.

Una de ellas tiene que ver con el "aprendizaje adecuado". Al respecto, la Unesco hace esta precisión: "En un mundo diverso, las necesidades de aprendizaje varían de una comunidad a otra, de modo que un aprendizaje adecuado deberá responder a lo que cada cultura, cada grupo humano, defina como necesario para vivir con dignidad" (2015, p. 33). De ser así, las herramientas para evaluar aprendizajes en entornos globales no necesariamente carecen de utilidad, pero tampoco tendrían que ocupar el papel central en el diseño y la valoración de proyectos educativos concretos y locales. Este es el punto de partida para nuestra investigación sobre capacidades para la agencia obtenidas a través de aprendizajes no académicos. En el caso del telebachillerato comunitario (TBC), hay evaluaciones estandarizadas nacionales que lo colocan en el nivel más bajo de desempeño académico; sin embargo, aún no se han identificado aprendizajes relevantes desde su propio horizonte comunitario. En esta problemática nos centraremos.

Dado que los TBC son relativamente recientes, no hay suficiente literatura sobre ellos. Los referentes principales hasta el momento (Weiss, 2017; Guzmán, 2018) retratan sus condidiones de precariedad; no obstante, cuando se realizaron aún no había egresados que pudieran dar cuenta de sus aprendizajes en retrospectiva. Las investigaciones sobre los TBC van en aumento, como se constató en el pasado Congreso Nacional de Investigación Educativa, en el cual hubo al menos cuatro ponencias sobre ellos desde diferentes áreas temáticas (Consejo Mexicano de Investigación Educativa, 2019); sin embargo, ninguna se refirió a aprendizajes no académicos y menos aun a sus egresados.

Para la elaboración de este artículo, nos basamos en datos de 2019 que provienen de una investigación sobre los recorridos y la capacidad de aspiración de los egresados del TBC en el estado de Morelos. Como parte de nuestro estudio, realizamos 30 entrevistas -25 de las cuales fueron con egresados-, pero en esa ocasión seleccionamos solo diez para el análisis. Ahora estamos retomando y examinando todas por completo, incluyendo las diez del estudio anterior. Esta vez nos enfocamos en responder la interrogante referente a las manifestaciones de aprendizajes no académicos, que incluyen la capacidad de aspiración y también van más allá de ella. Con esto, aportaremos a un primer acercamiento a la identificación de aprendizajes no 
académicos desde los TBC, que contarán por primera vez con un estudio de egresados desde esta perspectiva.

A continuación reseñamos los antecedentes que configuran la problematización abordada. Posteriormente, describimos los referentes teóricos y los criterios metodológicos que utilizamos. En otro apartado señalaremos los resultados obtenidos y nos apoyaremos en algunos ejemplos concretos. Por último, compartiremos las conclusiones más relevantes para esta investigación.

\section{ANTECEDENTES}

El TBC inició el sexenio pasado, en 2013, como un programa piloto que constituyó una estrategia del gobierno del presidente Peña Nieto para incrementar la cobertura en el nivel medio superior en poblaciones rurales alejadas de otras opciones escolarizadas, en un radio de al menos cinco kilómetros (Secretaría de Educación Pública [SEP], 2018). La apuesta por incrementar la cobertura del nivel medio superior provenía del sexenio del presidente Calderón, quien había impulsado primero una reforma curricular para todo este nivel educativo en 2008 (SEP, 2008) y, más tarde, en 2012, se le hizo parte de la educación obligatoria, con la meta de alcanzar la cobertura total en 2021 (Presidencia de la República, 2012). El modelo del TBC se retomó de otro que ya se aplicaba en Veracruz y proponía aprovechar durante las tardes las instalaciones de las telesecundarias (de ahí que se les llamó telebachilleratos); funcionarían de manera presencial con tres profesores, de los cuales uno fungiría además como responsable. Se les contrataría por honorarios y entre todos brindarían el 80\% de las horas previstas en el currículo (SEP, 2018), si nadie faltara nunca.

A estas circunstancias -aunadas a la precariedad de recursos estructurales-, se le suma el que el TBC tuvo el menor promedio de puntaje a nivel nacional en la prueba del Plan Nacional para la Evaluación de los Aprendizajes (Planea) más reciente, para el nivel medio superior, prueba que procura medir el desempeño académico en matemáticas, lenguaje y comunicación (Instituto Nacional para la Evaluación de la Educación, 2017).

Por otra parte, diversos actores relacionados con los TBC los han señalado como proyectos nobles con resultados valiosos para la formación de jóvenes -y no tan jóvenes-, así como para el desarrollo de sus comunidades, también en condiciones vulnerables o de marginación (Weiss, Antonio, Bernal, Guzmán y Pedroza, 2017); sin embargo, esto no se refleja en sus indicadores numéricos. En cambio, se recurre al término "precariedad" para referirse al pago de salarios de los docentes y su falta de contrato, a los planteles prestados que carecen de internet y, en ocasiones, también de agua o luz, a la economía de comunidades docentes y estudiantes, e incluso para describir las trayectorias académicas de los estudiantes (Guzmán, 2018; Weiss, 2017).

Con este panorama, nos propusimos revisar los casos de 25 egresados del TBC en el estado de Morelos a fin de rastrear sus principales aprendizajes no académicos y valorar su relevancia en términos de agencia desde una perspectiva de desarrollo humano.

Morelos es un estado con características particulares que reflejan de distintas maneras la realidad nacional. A pesar de que tiene apenas el 1.6\% de la población del territorio nacional, el promedio de años de escolaridad y los porcentajes de acceso a 
los niveles medio superior y superior son cercanos a los del nivel nacional (Instituto Nacional de Estadística y Geografía [INEGI], 2015). Asimismo, el peso de los estratos socioeconómicos se asemeja en forma considerable a los del país en su conjunto (Asociación Mexicana de Agencias de Inteligencia de Mercados, 2018). Todo ello nos aporta indicios de que, con base en un estudio de casos, los aspectos estructurales observados pueden plausiblemente repetirse en numerosos escenarios del país. Por otra parte, el tamaño del territorio morelense facilita el desplazamiento y acceso a comunidades apartadas, además de que otros indicadores demográficos, económicos, de salud, trabajo, vivienda y calidad de vida también se corresponden -guardando las debidas proporciones- con el conjunto nacional (INEGI, 2018).

Los TBC en Morelos se distribuyen en ocho planteles, de los cuales nuestra investigación incluyó entrevistados de cinco de ellos. Estos se encuentran en poblaciones consideradas de alta marginación (Secretaría de Desarrollo Social [Sedesol], 2013). En resumen, se trata de procesos educativos llevados a cabo en contextos de precariedad y con resultados oficiales poco alentadores. Ante ello, nuestra investigación parte teóricamente desde un enfoque de desarrollo humano y agencia.

\section{REFERENCIAS TEÓRICAS}

El enfoque de desarrollo humano es referido por Nussbaum (2012) como un enfoque de capacidades (capabilities) que aluden en específico al ser humano. Desde esta perspectiva, toma un papel central la libertad de cada persona para definir lo que ella misma pueda ser o hacer, de manera que la persona sea un fin y no sea considerada solo un instrumento para otro fin (por noble que parezca).

Los antecedentes de este enfoque se remontan a la necesidad de evaluar las condiciones y la calidad de vida de las personas y sus sociedades sin ceñirse solo a indicadores económicos, como el producto interno bruto, lo que gracias a la iniciativa de Amartya Sen dio lugar al Índice de Desarrollo Humano, adoptado por el Programa de las Naciones Unidas para el Desarrollo y que se refleja, hasta la fecha, en una serie de informes internacionales que iniciaron en 1990.

Para Sen (2019), el enfoque de capacidades también se centra en la libertad, entendida como las posibilidades reales que tiene una persona para lograr o conseguir algo que valora, y en ello basa su teoría de justicia, pues desde su perspectiva la distribución de capacidades es de mayor relevancia que la de bienes materiales, ya que de poco servirían las posesiones si con ellas no se pudiera obtener lo que se valora. De acuerdo con Nussbaum (2012), "el enfoque se ocupa de la injusticia y la desigualdad sociales arraigadas y, en especial, de aquellas fallas u omisiones de capacidades que obedecen a la presencia de discriminación o marginación" (p. 30).

Así, las capacidades son conjuntos de funciones con las cuales una persona puede elegir actuar para alcanzar fines elegidos por ella misma, y a los gobiernos les correspondería establecer las condiciones sociales para que esto sea posible, siempre que con ello no se actúe en detrimento de las capacidades más importantes de los demás.

Entonces, el enfoque de capacidades aporta una orientación específica que en este caso se aproxima más a una perspectiva antropocéntrica o humanista. También se trata de una aproximación coherente con los esfuerzos internacionales por 
replantear la educación expresados por la Unesco, la cual ha denunciado que, en aras de la economía, se ha utilizado a la educación para promover la producción irresponsable, que ha desembocado en una crisis ambiental (Unesco, 2015). Del mismo modo, se ha comprometido con el cuarto objetivo de desarrollo sustentable propuesto por las Naciones Unidas: “Garantizar una educación inclusiva, equitativa y de calidad y promover oportunidades de aprendizaje durante toda la vida para todos" (Unesco, 2016, p. 15).

Por otra parte, el enfoque reconoce la dignidad humana desde la agencia de las personas, entendiendo esta como una "capacidad de acción del individuo" (Nussbaum, 2012, p. 42); sin embargo, es preciso profundizar más en este concepto y su utilización dentro del ámbito educativo (Yurén y Mick, 2013). Cabría comenzar por especificar que la agencia en sí misma no es una capacidad, sino el ejercicio de diversas capacidades y se traduce en acción que transforma el contexto; entonces, más que hablar de "capacidad de agencia", en educación se debería hablar de capacidades para la agencia, y destacar que es menester que, efectivamente, pueda haber un cambio -fehaciente- en el contexto, que se pueda llevar a cabo y que su resultante sea deseada, elegida y asumida con responsabilidad por cada estudiante.

Esta concepción de capacidades y agencia parece contraponerse en diversos aspectos frente a los modelos basados en competencias, pues la agencia no responde de modo necesario a las "demandas" del entorno, sino a las de la persona-agente (quien idealmente tomaría en cuenta de manera crítica las circunstancias del entorno). La agencia tampoco considera satisfacer las expectativas de un gobierno, la industria, un colectivo o un tercero, ni ser inteligente para "solucionar" problemas a criterio de un cliente o usuario; más bien otorgar el poder -con su correspondiente carga ética- a la persona que elige actuar o dejar de hacerlo.

Sin embargo, ambas apreciaciones -competencias y capacidades- no son excluyentes entre sí y pueden coexistir si se establecen con claridad las prioridades del modelo (Millán y Martínez, 2017). Por ello, también cobra importancia contar con más referentes de valoraciones explícitas sobre la agencia y las capacidades que implica, pues así se podrían diseñar estrategias para favorecerla y empoderar a los actores que, como agentes, podrán modificar con mayor efectividad entornos de marginación y desigualdad, lo cual probablemente no sea indispensable en todos los escenarios educativos, pero sí en los más vulnerables.

Superar condiciones de pobreza es algo que ha sido trabajado desde la perspectiva antropológica por Appadurai (2013), quien estudió los esfuerzos colectivos de comunidades hindúes en grandes urbes, y encontró que su capacidad de voz y aspiración eran indispensables para que ellos mismos pudieran determinar los caminos más convenientes para superar estas condiciones, desde sus propios significados culturales.

A la capacidad de voz y aspiración se le podrían sumar otras habilidades y competencias a las que se les suele llamar "blandas" -de naturaleza no académicamás relacionadas con cuestiones actitudinales y de formación en valores que, de acuerdo con Pieck y Vicente (2017), son fundamentales para el éxito de los programas de capacitación para el trabajo en el nivel medio superior, en particular en 
el caso de jóvenes provenientes de sectores vulnerables; algunas de ellas son "ética, responsabilidad, empatía, espíritu de servicio, trabajo en equipo, sociabilidad, entre otras" (p. 389).

Somos conscientes de que este tipo de teorización, con nociones conceptuales poco desarrolladas, no profundiza en la descripción de cada uno de estos términos relevantes, como son "desarrollo humano", "capacidades", "agencia” o "capacidad de aspiración". No obstante, el criterio para su utilización es predominantemente pragmático y atiende, de acuerdo con Chinoy (1966), la consideración de que los conceptos en el ámbito sociológico se eligen y desarrollan más según su utilidad que con la pretensión de agotar su descripción compleja. En nuestro caso, buscamos que estas nociones contribuyan a una mejor comprensión de los aprendizajes no académicos de personas a quienes se reconoce su dignidad y capacidad de aspiración entre otras tantas más que les permitirían elegir con agencia cómo modificar su entorno conforme a sus propios intereses y los de sus comunidades; en este sentido, estos conceptos se relacionan con un proyecto educativo, si se les atribuye una finalidad como la referida por la Unesco (2015) al replantearse la educación.

¿De qué manera se esperaría poder dar cuenta de este tipo de aprendizajes en entornos en especial alejados, como son los del TBC? De ello nos ocuparemos en el siguiente apartado.

\section{Metodología}

Es conveniente diseñar las estrategias metodológicas con base en los planteamientos teóricos o epistemológicos previamente definidos, al menos en el ámbito de la investigación cualitativa (Vasilachis, 2006; Silverman, 2010). En nuestro caso, tomamos como punto de partida el enfoque de capacidades, que encierra nociones específicas sobre el ser humano, la libertad y la justicia. Esperamos que el camino metodológico sea congruente con ello; por eso, consideramos la pertinencia de fundamentarnos en la perspectiva fenomenológica.

En cuanto a aspectos clave en nuestra postura teórica, para el enfoque de capacidades, el ser humano -con agencia- es digno, lo que significa que tiene un valor intrínseco que impide tratarlo como medio o instrumento para algún fin, ya que es un fin en sí mismo; construir o abonar para su desarrollo individual y colectivo es la finalidad del enfoque y también lo sería para la educación. La noción de justicia supone el procurar un estado mínimo de bienestar para todos los seres humanos, que incluye el poder convivir con un medio natural sustentable. Del mismo modo, los seres humanos, en general, son capaces de desarrollar agencia y esta conlleva libertad, así como responsabilidad.

Para identificar y valorar los aprendizajes no académicos de personas que egresaron del nivel medio superior (específicamente del TBC), es pertinente partir desde su propia percepción o "experiencia vivida". Este es el eje de la aproximación fenomenológica. Por ello, nuestro camino metodológico comienza buscando

privilegiar el punto de vista de los actores aludidos y continúa involucrándose con su contexto personal, cultural y escolar. 
En nuestra investigación identificamos entre los casos las mayores similitudes y diferencias sobre cuáles son y cuál es la manera en que los participantes valoran sus principales aprendizajes no académicos. Entonces, la compatibilidad de la aproximación fenomenológica con el estudio de caso se justifica, porque la primera "intenta fundamentalmente explicar los significados tal como los vivimos en nuestra existencia cotidiana, en nuestro universo vital" (Van Manen, 2003, p. 29), mientras que el segundo es un método empírico cuya meta es comprender el caso para facilitar la investigación de fenómenos contemporáneos en profundidad cuando los límites entre el fenómeno y el contexto podrían no ser del todo claros (Yin, 2018).

Para estructurar este apartado metodológico, lo organizamos en tres categorías descriptivas para la vía metódica en investigaciones educativas con referentes empíricos (Alanís, 2015), que corresponden a los conceptos ordenadores en relación con los observables, a los instrumentos de recolección y soporte de datos, y a las herramientas analíticas. Con esto, pretendemos cubrir la información necesaria para valorar de manera intersubjetiva -y finalmente reproducir en otros escenarios- los pasos que seguimos en nuestra investigación.

El concepto ordenador se refiere al tema que se busca resaltar dentro de la experiencia vivida de las personas en quienes se centró esta investigación, es decir, los aprendizajes aportados por los TBC a sus egresados, en especial los aprendizajes no académicos, como el desarrollo de la capacidad de voz, la capacidad de aspiración y demás cuestiones actitudinales o de formación de valores, como las referidas por Pieck y Vicente (2017). Estos aprendizajes difícilmente son observables de manera directa, por lo que en la investigación recurrimos a referentes indirectos que ayudaron a dar cuenta de esto. Nuestra intención fue vincularlos con fragmentos discursivos de los actores participantes. Estos fragmentos fueron nuestros observables, pues de modo tangible constituyeron los datos empíricos de la investigación.

El diseño de la recolección de datos correspondió a un estudio de casos múltiples de unidades incrustadas, siguiendo la propuesta de Yin, quien la denomina "tipo 4" (Yin, 2018). Con base en este esquema, cada uno de los cinco planteles (seleccionados por su disponibilidad para participar en el estudio) representó un caso elegido desde una lógica de replicación literal, es decir, en función de sus similitudes más que de sus diferencias. Estas podrían caracterizarse de manera resumida: planteles con carencias de infraestructura, los cuales habían iniciado en instalaciones prestadas por una telesecundaria (solo uno ha conseguido mudarse luego a unas pequeñas instalaciones propias financiadas por la comunidad), con solo tres profesores (uno de ellos fungía a la vez como responsable) en condiciones de precariedad laboral (sin contratos, sueldos retrasados), con un solo grupo por grado, en localidades catalogadas con alto grado de marginación (Sedesol, 2013).

Dentro de cada caso o plantel, seleccionamos a participantes específicos o "unidades incrustadas", elegidos por su disponibilidad para la investigación, pero también en busca de satisfacer criterios de replicación teórica, es decir, poder contrastar diferencias entre subgrupos: hombres y mujeres, que estudian o trabajan (de manera remunerada o no), y con uno, dos o hasta tres años desde su egreso de la educación media superior. Para ello, procuramos, como sugiere Yin (2018), que se contara en cada caso con al menos dos integrantes por subgrupo. 
La Dirección General de Educación Media Superior y Superior del estado de Morelos solicitó por escrito a los responsables de plantel darnos acceso para realizar la investigación. Así, llevamos a cabo 25 entrevistas semiestructuradas en profundidad a fin de adecuarnos a las orientaciones de Hammer y Wildavsky (1990), con egresados de cinco de los ocho TBC que hay en Morelos, distribuidos conforme a la tabla.

Tabla. Distribución de egresados entrevistados por plantel

\begin{tabular}{|c|c|c|c|c|c|}
\hline Plantel & Hombres & Mujeres & Estudian & No estudian & Egresaron en \\
\hline A & 2 & 3 & 3 & 2 & 2016,2017 y 2018 \\
\hline B & 2 & 4 & 2 & 4 & 2016,2017 y 2018 \\
\hline C & 3 & 3 & 3 & 3 & 2016,2017 y 2018 \\
\hline D & 2 & 2 & 2 & 2 & 2017 y 2018 \\
\hline E & 2 & 2 & 1 & 3 & 2017 y 2018 \\
\hline Total & 11 & 14 & 11 & 14 & \\
\hline
\end{tabular}

Asimismo, entrevistamos a sus cinco responsables de plantel (dado que su nombramiento no es de "directores"). Las entrevistas iniciaron en junio de 2019 y terminaron en agosto de ese mismo año. La mayoría se llevó a cabo al interior de los mismos planteles. A los participantes les pedimos su consentimiento después de explicarles la finalidad de la investigación, así como las condiciones de confidencialidad y anonimato; todo ello quedó registrado en las mismas grabaciones, las cuales se hicieron con dispositivos digitales en formato mp3 con duración promedio de 59 minutos. Luego se respaldaron en Google Drive.

La guía de entrevista se dividió en tres secciones: introducción, desarrollo y conclusiones. En la primera, incluimos lo referente al consentimiento informado, datos generales de registro y preguntas de apertura. En el desarrollo, abordamos módulos de preguntas sobre su trayectoria escolar con especial atención en lo que les fue aportado por el TBC. Otro sobre su trayectoria laboral; un tercer módulo sobre sus actividades actuales y un cuarto acerca de perspectivas a futuro. Finalmente, en la sección de cierre les formulamos preguntas para determinar su nivel socioeconómico, un ejercicio de campos semánticos y datos de contacto.

Para el análisis de datos, convertimos todas las entrevistas a formato .wav monoaural de 8 bits a fin de procesarlas con el software de acceso abierto Sonal (Alber, 2018), que resultó de mucha utilidad porque nos permitió tematizar entrevistas conforme se les escuchaba. También, recurrimos al dictado de voz de Google Docs para elaborar una primera versión de las transcripciones totales de cada entrevista, las cuales corregimos para revisar su puntuación y subsanar otros errores. A principios de 2020, tematizamos todas las entrevistas para recuperar sus referencias concretas hacia aprendizajes no académicos.

Para la codificación temática, consideramos, junto con Flick (2007), que

el supuesto subyacente es que en mundos sociales o grupos diferentes se pueden encontrar distintas visiones. Para evaluar este supuesto y para desarrollar una teoría de las maneras de ver y experimentar específicas de estos grupos, es necesario modificar algunos detalles del procedimiento de Strauss para aumentar la comparabilidad del material empírico (p. 201). 
El modelo metodológico prevé una serie de casos y se comienza por producir descripciones breves de cada uno, etiquetarlas con una frase o lema representativo y agregarles una breve descripción de cada entrevistado, así como sus menciones relativas al tema investigado. A continuación, establecimos un sistema de categorías provisional y luego, en cada caso, realizamos una codificación abierta para después efectuar otra más de tipo selectiva, de manera similar a la propuesta de la teoría fundamentada (Strauss y Corbin, 2002), pero sin hacer hincapié en consolidar una categoría central, sino en identificar dominios temáticos para cada caso individual. Conforme se avanza, los nuevos casos se van comparando y ajustando en función de los primeros y viceversa, con lo que, al final, se consigue contar con una estructura apta para la comparación de casos y grupos (Flick, 2007).

En nuestra investigación, estos procesos se realizaron primero sobre las entrevistas de los egresados -a quienes les asignamos seudónimos provenientes de personajes de ficción- y luego agrupamos esta información por cada uno de los cinco planteles; tomamos en cuenta, además, los comentarios vertidos por los responsables de plantel. Posteriormente, la categorización se efectuó para cada caso ajustando y modificando las temáticas y categorías de modo progresivo. La síntesis de los resultados obtenidos se presenta en el siguiente apartado.

\section{RESULTADOS}

A pesar de que la guía de entrevista estuvo diseñada para caracterizar la capacidad de aspiración y su entorno cultural, en diversos momentos hubo comentarios que hicieron alusión a diferentes aprendizajes -o a sus efectos- de carácter no académico. Como señalan Strauss y Corbin (2002), no es recomendable la rigidez en la codificación procedente del análisis, pues los sucesos que se codifican de cierta manera en un caso, bien pueden codificarse de forma distinta en otro. En nuestra categorización, llegamos a asignar expresiones similares -o en ocasiones iguales- dentro de categorías distintas, dada la influencia de cada caso y el contexto sobre las interpretaciones y sus implicaciones. Cabe señalar que tanto los nombres de las categorías como sus códigos subyacentes se formaron con terminología propia del lenguaje del análisis, más que de las expresiones directas de los entrevistados.

Una vez advertida esta eventualidad, señalamos que las entrevistas se filtraron buscando fragmentos con indicios de aprendizajes no académicos. En ellos encontramos una gran diversidad de expresiones que agrupamos en cuatro categorías en función de la agencia: autonomía, amplitud, efectividad y orientación. Ciertamente, estas categorías se definieron con base en agrupaciones de códigos, pero, de acuerdo con la metodología de análisis, estas familias de códigos se fueron modificando y enriqueciendo conforme se analizaban, de manera que la inclusión final de códigos bajo cada categoría fue lo bastante extensa como para plasmarla en este espacio.

Por otra parte, dado que se han respetado las condiciones de confidencialidad y anonimato, no consideramos necesario volver a contactar a los participantes para informarles los resultados preliminares de la investigación; sin embargo, sí tenemos previsto regresar a los TBC participantes a comentar los hallazgos y las conclusiones de este estudio una vez que las actuales condiciones de confinamiento por la pandemia del covid-19 lo permitan. 


\section{Autonomía}

En esta faceta identificamos los aprendizajes que provocan cambios significativos dentro de las personas, sobre todo de lo que piensan de sí mismas, es decir, saber quiénes son (autopercepción, identidad), lo que creen que merecen y pueden lograr (autoestima, autoconfianza), y si saben lo que quieren o hacia dónde quieren ir (autodeterminación). Por eso, hemos llamado a todo junto "autonomía", que encuentra su raíz en la persona que se puede normar a sí misma, y establecer sus propias leyes y límites que, en última instancia, le hacen capaz de elegir en quién se convertirá en medio de sus circunstancias.

En ocasiones, los mismos egresados se dan cuenta de esto, y también lo llegan a apreciar los responsables de plantel: "Ya después ellos solitos van tomando sus propias decisiones, y veo que efectivamente les ayuda a tener esa confianza en sí mismos, de que sí pueden, de que sí pueden. Y se me hace eso, pues, padrísimo, ¿no? en donde uno interviene para lograr esa seguridad en ellos" (R1).

De alguna manera, esta confianza en que las cosas para ellos y para otros a su alrededor pueden mejorar, la convicción de que "otro mundo es posible", está también relacionada con la motivación para identificar nuevos horizontes deseables para ellos mismos y, con esto, se despierta su capacidad de aspiración: "Pues a mí el bachillerato me hizo diferente, porque así yo sé lo que yo quiero” (Eugene).

Este tipo de experiencias de empoderamiento trasciende las capacidades físicas para terminar influyendo sobre la manera en que los egresados ahora se ven a sí mismos, con lo que se activan sus aspiraciones: “... a mí me hizo crecer mucho, mucho, mucho como persona, como líder [...] yo llego y a lo que venga, a trabajar, sí, la verdad sí, y pues creo que es un proyecto que nos ha favorecido a muchos" (Kara).

Como apreciamos en el fragmento anterior, hay una nueva forma de posicionarse ante los demás. En el caso de Kara, ella se ve a sí misma como una líder. Estudia Ingeniería de Negocios y avanza con mucha claridad hacia un trabajo empresarial como paso intermedio hacia su propia compañía con espíritu emprendedor. No obstante, este empoderamiento y capacidad de aspiración, aunque puede percibirse más claramente en quienes continuaron sus estudios, también se ve reflejada (aunque con mayor palidez) en quienes no lo hicieron. Es el caso de Gretchen, quien, por circunstancias familiares, no ha podido continuar estudiando en el nivel superior, pero que tuvo una gran experiencia concursando y siendo premiada a nivel estatal: “... hemos salido del telebachillerato y quizás somos capaces de hacer más cosas que las otras escuelas, me refiero a mi caso, que siendo de un pueblo y de una escuela que apenas inició llegamos a metas, así que sí se puede mientras uno quiera seguir estudiando, se pueden alcanzar las metas" (Gretchen).

Incluso, en casos en que no había muchas opciones alentadoras en el futuro, el paso por el TBC se reconoció como un espacio de crecimiento personal y maduración que permite adentrarse en la adultez con mejores herramientas personales, como lo expresa esta pareja que se casó tras egresar:

Pedro: Como que te pone a madurar más, ya dejas de ser el niño que eras en la primaria, secundaria y ahorita en el bachillerato ya puedes ver más o menos a lo que le vas a tirar ya cuando eres mayor, sí. 
Wilma: Sí, dejas de actuar como una niña sin sentido. Ahora piensas como mujer, ahora ves que tienes un futuro adelante...

Amatista, otra entrevistada, no tiene intenciones próximas de formar una familia e ingresó al TBC después de trabajar por su cuenta siete años en una lavandería y como empleada doméstica, de ahí que, al iniciar sus estudios en el telebachillerato, ya tenía una perspectiva adulta. Ahora estudia Derecho: “... como que no sólo te están preparando para que salgas y sepas todo, sino también para que seas una mejor persona y mejor ser humano; te están preparando para que te concientices [...] la percepción que yo tengo del telebachillerato es como esa oportunidad de dejar de ser la persona que eras, o sea, ser alguien mejor".

Los cambios al interior no solo impactan la imagen de sí; a veces con ello también se modifica la perspectiva sobre la manera de relacionarse entre géneros:

Investigador: ¿Cuáles dirías aquí que fueron tus principales aprendizajes?

Molly: Pues el principal, el principal, fue que hubo igualdad de género. Que aquí no porque seas una señora te hicieron menos. 0 sea, fue parejo. Eso es lo que yo aprendí aquí. [...] y a ser un poquito, un poquito más este [...] ser este [...] tener autoestima; de que yo puedo. Ser mujer, pues.

No fueron pocos los comentarios que hicieron alusión al machismo, y fue notorio que estos procesos de cambio de mentalidad respecto al género no se quedan únicamente dentro de los TBC.

Finalmente, pero no de menor importancia, es el aprendizaje que se traduce en interés por seguir aprendiendo, aun cuando las opciones para hacerlo sean tan limitadas: "Sí lo volvería a hacer. Sí, ahorita ya se me quitó el miedo, eso ya pasó y sí lo volvería a hacer. Creo que sí me interesaría mucho aprender más cosas, nuevas cosas" (Helen).

\section{Amplitud}

Esta se refiere a la cantidad de opciones de acción a las que se puede acceder, vinculada, en este caso, a los aprendizajes no académicos. A mayor cantidad de opciones y funcionamientos, mayores posibilidades de modificación del contexto de acuerdo con las intenciones de la persona agente. En este rubro, descartamos los aprendizajes académicos presentes en el plan de estudios del nivel medio superior, así como los conocimientos técnicos utilizados en el trabajo, puesto que consideramos, de manera explícita, que esto es parte de sus objetivos formativos. Descartamos, así, los aprendizajes de actividades económicas y técnicas productivas que habían sido mencionadas con frecuencia.

Aun así, el rubro contó con numerosas alusiones a cualidades intangibles aprendidas o reforzadas en el TBC, pues incluimos aquí todas esas habilidades útiles para la ciudadanía, entre las cuales sobresalió la capacidad de voz, habilidades para conocer, relacionarse y convivir con más gente, así como con el medio ambiente, controlar emociones, superar o manejar situaciones difíciles: "Saber cómo canalizar o actuar frente a casos de violencia intrafamiliar, y otros problemas que tienen los jóvenes, alcoholismo, drogadicción, embarazos" (R1), adicciones, identificar problemas y so- 
lucionarlos, aplicar conocimientos a su vida práctica en casa, capacidad para ver más allá, salir de su comunidad y, finalmente, flexibilidad mental para cambiar de perspectiva, ver diferentes facetas en los problemas y crearse nuevos escenarios.

Veamos ahora algunos ejemplos: "Algo que me dejó muy bien aquí el telebachillerato y nunca se me va a olvidar es esa frase de que siempre viéramos a los problemas como nuevas oportunidades de vida, y creo que esa frase la he tenido siempre, siempre, siempre en mi mente y siempre que tengo un problema recuerdo esa frase; nos lo decía una maestra de aquí" (Kara).

En esta faceta ya no se trata de verse a sí mismo de modo diferente, sino de ver al mundo de otra manera y así identificar más posibilidades de acción. En el fragmento anterior constatamos cómo, más allá de las habilidades adquiridas, la capacidad de ver los problemas desde otra perspectiva los transforma en nuevas oportunidades para elegir y actuar: "Yo creo que todo sirve, empezando con la materia de ciencias de la comunicación [...] porque si no hay comunicación uno no puede moverse en la vida" (Gretchen). La "Voz", como la llama Appadurai, tiene diversas manifestaciones y en esta faceta se ubicaron formas diversas y, a la vez, más recurrentes para expresarse. En el caso del fragmento al inicio de este párrafo, se relaciona con la posibilidad de "moverse en la vida". Otros casos similares hacen referencia a la capacidad de voz que vence la "pena”: “... a través de esos proyectos que hicimos perdimos la pena, igual en los trabajos que hacíamos; en la exposición supimos cómo hablar, la forma de cómo hablar hacia la gente, perdimos la pena, el miedo de presentar un trabajo en PowerPoint, exponer" (Silver).

Otro modo de referirse a la capacidad de voz se manifiesta en la manera de relacionarse con otras personas y esto, a su vez, puede abrir nuevas posibilidades: “... el maestro Emmanuel me daba consejos, me decía que era buena para eso, pero yo siempre fui como que [...] no sé, soy muy penosa, entonces perdí el miedo a hablar ante la gente porque hasta exponer te ayudaba, todo el tiempo" (Eep).

A menudo, esta capacidad viene emparejada con nuevos esquemas mentales a los que se suele describir como "abrir los ojos", lo que es significativo si consideramos que en esta faceta se incluye el identificar nuevas oportunidades:

Investigador: ... sobre las cosas que tú sientes que te aportó el bachillerato.

Helen: Sí, pues eso, es que antes era yo así, muy sumisa, no hablaba, no decía lo que pensaba y pues con esta experiencia que tuve pues sí me ayudó mucho. Aprendí muchas cosas; ya parezco perico, ijajaja! Y pues sí, la verdad que no me arrepiento de haber tomado esta decisión y me ayudó mucho. Sí, me abrió los ojos. Sí.

Otro tipo de opciones vinculadas a una forma de ver diferente, pudimos identificarlo en la apropiación de una visión sustentable: "Yo reciclo el agua de lluvia para lavar" (Arya), y sobre todo en transformaciones provenientes de procesos de inculturación, en el sentido más amplio de la palabra, como se refleja en el comentario de esta responsable: “... nadie los había sacado, o sea, ellos no conocían más que el pueblo o sus comunidades, pero más allá ya no. Entonces algo que tuvimos fue una convivencia en un cine, todos los telebachilleratos y nos los llevamos, entonces pues así como que [...] ¡no habían ido al cine!” (R3). 
Finalmente, muchos aprendizajes que estaban destinados a servir como capacitación para el trabajo pueden ir mucho más allá, cuando los egresados se dan cuenta de que con esos mismos conocimientos pueden modificar sus propias condiciones de vida, y este metaaprendizaje es también una ganancia en términos de amplitud de agencia: "Y eso pues sí ayuda muchísimo; lo de la electricidad en la casa ¿no? y digo, pues creo que va creciendo ¿no? y creo que se va aprendiendo más” (Helen).

\section{Efectividad}

Molly: Pues es que la mayoría de aquí del pueblo, pues toma mucho. Raro es el que estudia; raro es el que termina.

Investigador: Cuando entran y estudian, ¿mejoran?

Molly: Pues yo creo que sí, porque [...] no al $100 \%$, pero sí se podría decir que un $50 \%$ están dedicados a estudiar y dejan un poquito el vicio, se podría decir.

Muchas veces, para que una persona pueda fungir como agente, no basta tener confianza en sí misma y más posibilidades de actuar; también es necesario corroborar que se pueden alcanzar los resultados esperados tras una elección. A esto se refiere Sen como "poder efectivo" (1985, p. 208) y se puede dar aun cuando el agente no tenga control sobre el procedimiento, pero sí sobre el desenlace. En el caso de los egresados del TBC, hubo aprendizajes no académicos que se tradujeron en mayor poder efectivo, como el logro de evitar una adicción (y no solo actuar frente a ella, como se mencionaba en la faceta anterior), evitar ser engañada, no darse por vencidos y defender sus derechos.

A estos aprendizajes específicos se les pueden sumar muchos otros que, aunque ya han sido mencionados, cobran un matiz diferente cuando su contexto hace resaltar el desarrollo o perfeccionamiento de una habilidad -como en la capacidad de voz-, pero que redunda en que con esta mejoría ellos pueden obtener resultados más precisos o mejores. Así lo manifiesta una responsable de plantel: "Y también algo de los egresados es que cuando se proponían algo, lo lograban, pero en equipo. [...] entonces, es algo que también el telebachillerato les ha enseñado; a que ellos pueden lograr cosas en equipo" (R3).

Por otra parte, la característica más reiterada en este aspecto de la agencia fue el aprender a no rendirse y perseverar, en especial porque "sí se puede". Este es, quizás, el aspecto más criticado desde el llamado "echaleganismo" (Krozer, 2019); sin embargo, para estos egresados su significado adquiere otro sentido. No hay que olvidar que las entrevistas partieron de una aproximación fenomenológica:

Arya: El último día de la graduación fue muy bonito, todos los consejos que nos dio la profesora.

Investigador: ¿Te acuerdas de alguno?

Arya: Que no nos diéramos por vencidos, que siguiéramos.

Algo similar mencionó otra egresada, madre soltera, trabajadora doméstica con dos hijos, de los cuales uno ya entró al TBC. Ella ya no pudo continuar estudiando, pero su aprendizaje se refleja en este consejo que da a los nuevos egresados: 
Helen: Sí, yo les recomendaría que no se trunquen en esto, que le echen muchas ganas. Sí, porque está muy difícil la vida ahorita. Si están deprimidos o que no encuentran algo, que se metan en los libros; los libros son muy buenos y nos abren mucho la mente. Sí, y así nos aprendemos a defender y más que nada a vivir, porque si aprendemos, estamos preparados, y que sigan echándole ganas hasta más no poder.

Cuando este discurso de "echarle ganas" además viene acompañado del compromiso de quien lo predica, la interpretación de quienes lo reciben se vuelve más significativa: “... los maestros siempre nos decían: 'No te salgas, échale ganas, tú puedes' y 'cualquier cosita, te podemos ayudar; si no entiendes la materia nos podemos quedar horas extras y te explicamos para que entiendas'” (Marge).

A diferencia de la capacidad de aspiración que notoriamente está más desarrollada en quienes lograron continuar con sus estudios, esta arenga para "echarle ganas" fue bien recibida tanto entre quienes sí siguieron estudiando como entre quienes no lo hicieron. Eep, por ejemplo, estudia enfermería y quiere ejercerla en Estados Unidos, que es donde ella creció:

Eep: ... le tenía mucho miedo a las agujas.

Investigador: $\mathrm{Y}$ ahora estudias para enfermera...

Eep: ... jajaja. Sí, siempre me decían: “¡Sí puedes!”.

En ese mismo plantel, tenemos el caso de Todd, quien después del TBC ya no siguió estudiando porque espera algún día heredar la carnicería de sus abuelos:

Investigador: ¿Qué dirías que te aportó tu bachillerato?

Todd: Pues sí me aportó mucho [...] sí, pues le digo, no rendirme en mi trabajo, ellos me enseñaron que siguiéramos adelante. La verdad no pude seguir estudiando por la economía, pero si algún día se me da, lo voy a hacer.

\section{Orientación}

Nuestra última categoría o faceta de la agencia involucrada en aprendizajes no académicos fue la última en ser delineada durante el análisis; sin embargo, su contenido hace referencia a cuestiones trascendentes que, para los agentes, cargan las acciones de sentido y hacen que estas valgan la pena. En este rubro destacan, principalmente, los valores -ya elegidos por los egresados- y con ellos la intención de ver por el bien de su comunidad de origen, aun cuando pudieran estar viviendo lejos de ella:

Investigador: ¿Qué les trajo el bachillerato a ellos, qué les dio?

Responsable 3: Pues yo siento que su conocimiento. Aunque estén de albañiles ellos saben ya su manera de desenvolverse, es muy diferente. Su respeto y todo lo que ellos tienen [...], su compromiso y responsabilidad de ellos.

Entre egresados se enseñan este tipo de aprendizajes, y muchas veces son los mismos compañeros quienes refuerzan actitudes y valores, como lo menciona Molly, que ingresó al TBC ya siendo adulta y con tres hijos: "Gracias a estos chicos aprendí a que pues hay igualdad ¿no? Tanto tienen derechos ellos como hombres, tanto como 
mujer, tenemos los mismos derechos". Del mismo modo, hubo casos en los que estos aprendizajes acompañaron a los temas del currículo, pero a veces ocurre que se aprende mucho más de lo que se pretende enseñar:

... Yo me quedo con eso, como de ver cómo alguien, un ser humano que ni te conoce ni eres de su familia, y que le están pagando por hacerlo, pero a pesar de que le están pagando, él está entregando lo mejor de sí ¿no? A él le costó muchos años estudiarlo y aprenderlo, y te lo está dando; te lo está regalando [...] Entonces como que con eso me quedo [...] Como el ver la pasión con la que a veces algunas personas hacen su trabajo (Amatista).

Uno de los valores más citados en este sentido fue la responsabilidad; también hubo otros que se emparejaron con intereses comunitarios:

Investigador: ¿Qué fue lo que te dejó más el telebachillerato?

Eep: Yo creo que unidad. [...] amor y lealtad, yo creo.[...] en saber que hay personas que necesitan más salud, tienes que encontrar la manera de ayudarlos igual beneficiándote a ti y a ellos; a ser más caritativos digámoslo así, a ver más por la comunidad, buscar problemas y solucionarlos.

Este arraigo a su comunidad puede ser conscientemente procurado por los profesores y responsables de plantel, quienes, en casi todos los casos observados, provenían de fuera de la comunidad. Sin embargo, su insistencia deja entrever el sistema de valores que va permeando en los estudiantes: “... por lo menos se les despierta el interés de cuidar el medio ambiente [...] están los estudiantes creando conciencia y teniendo amor e interés por su comunidad, que es el objetivo" (R6).

Cuando estos discursos llegan a los estudiantes, no siempre parece que ellos les ponen atención, pero con el egreso viene una revaloración de experiencias de las que ellos pueden extraer una noción de sentido para motivar y orientar sus acciones: “... pero sí, el telebachillerato para mí fue lo máximo, porque te deja ser tú, además de que también es 'comunitario', es decir, que siempre teníamos que ver cómo ayudar a la gente, siempre eso nos decían: ‘ ¿cómo ayudar a la gente? Es comunitario. Tienen que ver por su comunidad; no solo ahorita, sino más adelante” (Kara).

El ver por el otro, el ser sensible ante las necesidades de los demás, conlleva una actitud que no solo se puede proyectar sobre las personas, sino sobre otros aspectos del entorno, incluyendo, desde luego, una orientación hacia la sustentabilidad: "Aquí mis principales aprendizajes [...] fue el cuidar el medio ambiente [...] y también me enseñaron bastante de humanidad" (Santo).

Es importante destacar que estas expresiones no son generalizadas. Como parte del análisis de casos, consideramos la búsqueda de contrastes; así, encontramos a quienes no mencionaron aprendizaje alguno que les fuera significativo. Otros más simplemente apelaron a lo inefable:

Investigador: ¿Te sirvió para algo?

GJazz: Para aprender cosas.

Investigador: ¿Igual algo que apliques?

GJazz: A lo mejor sí, pero no sabría cómo decirle. 


\section{CONCLUSIONES}

Al indagar sobre aprendizajes no académicos, surgen infinidad de nuevas preguntas en muchos sentidos. Ello nos obliga a buscar identificar primero las más relevantes y asequibles para ser contestadas y, por ello, esta investigación se planteó partir de identificar dichos aprendizajes y valorar su relevancia desde un enfoque de agencia y desarrollo humano como el referido por Sen y Nussbaum.

Los indicadores cuantitativos con los que más se suele trabajar (como los resultados de Planea o los promedios de calificaciones en los certificados) son cuestionados en tanto que no reflejan las necesidades ni responden a las demandas de capacidades centrales para las personas involucradas en estos procesos formativos ni sus comunidades. Sus resultados solo pueden ser útiles si se considera su contexto, así como la finalidad que se proyecta para su educación, como la que señala la Unesco.

Cualquier evaluación tendría que ser congruente con estos factores, pero si nos basamos en ellos y en las apreciaciones de los actores involucrados de manera directa, desde sus comunidades y lo que es observado en sus planteles, encontramos que diversos tipos de conocimiento entran en juego para posibilitar que estas personas cuenten con mejores herramientas para construir su propia historia y lidiar con sus retos individuales y colectivos en sus términos, como lo refiere Appadurai (2013). También observamos que muchos de estos aprendizajes no están previstos en el currículo.

Desde un enfoque de desarrollo humano identificado con capacidades, señalamos la importancia de adquirir funcionamientos para alcanzar fines libremente elegidos. Con estas capacidades se construye agencia -que implica cambios en el contexto-, en función de la cual se reconoce la dignidad humana. La agencia permite a las personas adueñarse de su proyecto vital y vislumbrar vías para la superación de condiciones de pobreza o marginación en términos elegidos por los mismos actores involucrados; así, contribuye a condiciones de mayor justicia social y se orienta también al cumplimiento de las finalidades de la educación.

Con esta investigación constatamos de qué manera la agencia se alimenta de capacidades como el uso de la voz o la capacidad de aspiración, entre muchas otras, a través de la mirada de la persona sobre sí misma, el reconocimiento de sus posibilidades, el logro de las resultantes deseadas y el sentido atribuido por el actor.

De nuestro estudio de casos múltiples incrustados, surgieron cuatro categorías correspondientes a facetas de la agencia adquirida por los egresados tras su paso por el TBC: autonomía, amplitud, efectividad y orientación. Cada una comprende variantes de aprendizajes no académicos y, en muchas ocasiones, no planeados ni esperados, los cuales, sin embargo, aportaron capacidades y agencia a los egresados.

Al comparar casos múltiples incrustados, identificamos que estos aprendizajes no son uniformes en ningún plantel. Siempre hubo quien los hizo conscientes en gran cantidad y de manera muy significativa, así como quienes no manifestaron ninguno. La proporción o gradualidad entre ambos extremos no es clara ni homogénea; no obstante, sí es notorio el reconocimiento y la gratitud de los egresados hacia su TBC; en todos los planteles encontramos que se desarrolló la agencia de los egresados desde todas sus facetas. Estos hallazgos nos brindan otros elementos para valorar su desempeño y la importancia de los aprendizajes 
que ahí se germinan; una valoración que desde la perspectiva de los egresados redunda en el mejoramiento de sus comunidades y de ellos mismos por medio de su agencia y adquisición de capacidades.

Así, apoyados en las percepciones y necesidades de los egresados, consideramos que los TBC tienden a criterios de excelencia no convencionales y, si bien tienen muchos aspectos en los que pueden mejorar -que también fueron profusamente señalados por los egresados-, representan un paso importante hacia una mejor educación para mucha gente que se encuentra en situación de vulnerabilidad.

Consideramos que estos aprendizajes, que fueron objeto de nuestra investigación, coadyuvan de manera muy importante a la consecución de las finalidades de la educación para las personas involucradas, y sin ellos estas finalidades habrían quedado fuera del alcance para los egresados, sus comunidades y nuestra sociedad, de modo que son imprescindibles. Por ello, es imperativo hacer estos señalamientos con carácter prescriptivo:

- Los resultados de evaluaciones estandarizadas deben ponerse en contexto y servir como herramienta para identificar los escenarios donde se requieren más recursos y, sobre todo, no deben impedir valorar los aprendizajes que, más allá de criterios académicos, aportan herramientas indispensables para el desarrollo de capacidades y agencia de los actores involucrados.

-El servicio de telebachillerato comunitario debe ser apoyado con mayor contundencia, en virtud de los beneficios que aporta y el papel que juega en el desarrollo de las comunidades más apartadas y vulnerables. Sus profesores deben ser puntualmente remunerados y su trabajo, reconocido con términos laborales estables; apoyarlos también con infraestructura, seguridad social y recursos para su formación continua.

- La investigación debe colaborar más con poner a prueba distintos modelos para el fomento de capacidades y agencia en los planteles educativos; corroborar la consistencia de estos hallazgos en otros escenarios y hacer los señalamientos que correspondan para incidir con efectividad en la transformación de la realidad social del país.

Queda pendiente identificar a qué obedece el que haya quienes estén desarrollando su agencia mientras que para otros el impacto de los TBC no alcanza a detonar este proceso con contundencia. Nuestro referencial teórico por sí solo no logra explicarlo y haría falta mayor investigación sobre este aspecto.

Finalmente, trascendiendo las improntas de una mirada crítica, es motivador y desafiante a la vez encontrar espacios como los TBC y constatar la forma en que los procesos educativos van haciendo posible que los egresados puedan transformar sus vidas de acuerdo con su propio proyecto. Aunque es cierto que todavía enfrentan muchas carencias y dificultades, lo que está sucediendo en estos planteles es también un paso esperanzador en la dirección correcta. 


\section{REFERENCIAS BIBLIOGRÁFICAS}

Alanís, J. F. (2015). Categorías descriptivas para la vía metódica en investigaciones educativas a partir de referentes empíricos. Ponencia presentada en el XIII Congreso Nacional de Investigación Educativa, Chihuahua, México. Recuperado de http://www.comie.org.mx/congreso/memoriaelectronica/v13/ doc/0064.pdf

Alber, A. (2018). Presentation of the software program SONAL. Bulletin of Sociological Methodology, vol. 137-138, núm. 1, pp. 176-200. doi: $10.1177 / 0759106318761613$

Appadurai, A. (2013). The future as cultural fact. Essays on the global condition. Londres: Verso.

Asociación Mexicana de Agencias de Inteligencia de Mercados (2018). Cuestionario para la aplicación de la regla AMAI 2018 y tabla de clasificación. Recuperado de http://nse.amai.org/wp-content/uploads/2018/04/CuestionarioNSE-2018.pdf

Chinoy, E. (1966). Introducción a la sociología. Buenos Aires: Paidós.

Consejo Mexicano de Investigación Educativa (2019). Memoria electrónica del Congreso Nacional de Investigación Educativa. Recuperado de http://www. comie.org.mx/congreso/memoriaelectronica/v15/index.htm

Flick, U. (2007). Introducción a la investigación cualitativa. Madrid: Morata.

Guzmán, C. (2018). Avances y dificultades en la implementación del marco curricular común. Telebachillerato estatal, educación media superior a distancia y telebachillerato comunitario. México: Instituto Nacional para la Evaluación de la Educación.

Hammer, D. y Wildavsky, A. (1990). La entrevista semiestructurada de final abierto. Aproximación a una guía operativa. Historia y Fuente Oral, núm. 4, pp. 23-61. Recuperado de http://www.jstor.org/stable/27753290

Instituto Nacional de Estadística y Geografía (INEGI) (2018). Morelos (17). Recuperado de https://www.inegi.org.mx/app/estatal/?ag=07000017\#grafica

INEGI (2015). Características educativas de la población. Recuperado de http:// www.beta.inegi.org.mx/temas/educacion/

Instituto Nacional para la Evaluación de la Educación (2017). Planea. Resultados nacionales 2017. Educación media superior. Lenguaje y comunicación. Matemáticas. México.

Krozer, A. (27 de agosto de 2019). La mentira de la meritocracia: para ser rico hay que nacer rico [entrada en un Blog]. Recuperado de https://economia. nexos.com.mx/?p=2496

Millán, E. y Martínez, C. (2017). Antes y después de la educación por competencias: aproximación crítica desde las capacidades humanas. En J. A. IbáñezMartín y J. L. Fuentes. Actas del VIII Congreso Internacional de Filosofía de la Educación (pp. 113-125). Madrid: Dykinson.

Nussbaum, M. C. (2012). Crear capacidades [versión Google Play Books]. Recuperado de https://play.google.com/books/reader?id=KyFj8PHvLU4C

Pieck, E. y Vicente, M. R. (2017). Abriendo horizontes. Estrategias de formación para el trabajo de jóvenes vulnerables. México: Universidad Iberoamericana. Recuperado de https://ibero.mx/web/filesd/publicaciones/abriendohorizontes.pdf 
Presidencia de la República (9 de febrero de 2012). Decreto por el que se declara reformado el párrafo primero; el inciso c) de la fracción II y la fracción V del artículo 3o., y la fracción I del artículo 31 de la Constitución Política de los Estados Unidos Mexicanos. Diario Oficial de la Federación. Recuperado de http://dof.gob.mx/nota_detalle.php?codigo=5233070\&fecha=09/02/2012

Secretaría de Desarrollo Social (Sedesol) (2013). Catálogo de localidades. Recuperado de la página web del Sistema de Apoyo para la Planeación del PDZP: http://www.microrregiones.gob.mx/catloc/Default.aspx?tipo=clave\&campo $=$ mun \&valor $=17$

Secretaría de Educación Pública (SEP) (2018). Documento base 2018. Adopción del modelo educativo para la educación obligatoria en el telebachillerato comunitario. México. Recuperado de https://www.dgb.sep.gob.mx/servicioseducativos/telebachillerato/normatividad/Documento-base-2018.pdf

SEP (26 de septiembre de 2008). Acuerdo número 442 por el que se establece el Sistema Nacional de Bachillerato en un marco de diversidad. Diario Oficial de la Federación, Recuperado de http://dof.gob.mx/nota_detalle.php?codigo $=5061936 \&$ fecha $=26 / 09 / 2008$

Sen, A. (2019). La idea de la justicia [versión Google Play Books]. Recuperado de https://play.google.com/books/reader?id=OLjUGtgDEOkC

Sen, A. (1985). Well being, agency and freedom: The Dewey lectures 1984. The Journal of Philosophy, vol. 82, núm. 4, pp. 169-221. doi: 10.2307/2026184

Silverman, D. (2010). Doing qualitative research (3 $3^{\mathrm{a}}$ ed.). Londres: Sage.

Strauss, A. y Corbin, J. (2002). Bases de la investigación cualitativa. Técnicas y procedimientos para desarrollar la teoría fundamentada. Medellín: Universidad de Antioquía. Recuperado de https://diversidadlocal.files.wordpress. com/2012/09/bases-investigacion-cualitativa.pdf

Organización de las Naciones Unidas para la Educación, la Ciencia y la Cultura (Unesco) (2016). Educación 2030. Declaración de Incheon y marco de acción para la realización del Objetivo de Desarrollo Sostenible 4. Recuperado de https://unesdoc.unesco.org/ark:/48223/pf0000245656_spa.locale=es

Unesco (2015). Replantear la educación. ¿Hacia un bien común mundial? París. Recuperado de https://unesdoc.unesco.org/ark:/48223/pf0000232697

Van Manen, M. (2003). Investigación educativa y experiencia vivida. Ciencia humana para una pedagogía de la acción y la sensibilidad. Barcelona: Idea Books.

Vasilachis, I. (2006). La investigación cualitativa. En I. Vasilachis. Estrategias de investigación cualitativa (pp. 1-22). Barcelona: Gedisa.

Weiss, E. (2017). Estudio exploratorio del modelo de telebachillerato comunitario y su operación en los estados. México: Instituto Nacional para la Evaluación de la Educación.

Weiss, E., Antonio, L., Bernal, E., Guzmán, C. y Pedroza, P. (2017). El telebachillerato comunitario. Una innovación curricular a discusión. Revista Latinoamericana de Estudios Educativos, vol. XLVII, núm. 3-4, pp. 7-26.

Yin, R. K. (2018). Case study research and aplications: Design and methods (6a. ed.) [versión Kobo epub]. Thousand Oaks: Sage. Recuperado de https://www. kobo.com/us/en/ebook/case-study-research-and-applications

Yurén, T. y Mick, C. (coords.) (2013). Educación y agencia. Aproximaciones teóricas y análisis de dispositivos. México: Juan Pablos. 